Diffraction grating eigenvector for translational and rotational motion

M. C. Rushford, W. A. Molander, J. D. Nissen, I. Jovanovic, J. A. Britten, C. P. J. Barty

July 30, 2005

Optics Letters 
This document was prepared as an account of work sponsored by an agency of the United States Government. Neither the United States Government nor the University of California nor any of their employees, makes any warranty, express or implied, or assumes any legal liability or responsibility for the accuracy, completeness, or usefulness of any information, apparatus, product, or process disclosed, or represents that its use would not infringe privately owned rights. Reference herein to any specific commercial product, process, or service by trade name, trademark, manufacturer, or otherwise, does not necessarily constitute or imply its endorsement, recommendation, or favoring by the United States Government or the University of California. The views and opinions of authors expressed herein do not necessarily state or reflect those of the United States Government or the University of California, and shall not be used for advertising or product endorsement purposes. 


\title{
Diffraction grating eigenvector for translational and rotational motion
}

\author{
Michael C. Rushford, William A. Molander, James D. Nissen, Igor Jovanovic, \\ Jerald A. Britten, and C. P. J. Barty \\ Lawrence Livermore National Laboratory, \\ Mail Code L-459, 7000 East Ave, Livermore, California 94550 \\ rushford1@,llnl.gov
}

Future energy scaling of high-energy chirped-pulse amplification systems will benefit from the capability to coherently tile diffraction gratings into larger apertures. Design and operation of a novel, accurate alignment diagnostics for coherently tiled diffraction gratings is required for successful implementation of this technique. An invariant diffraction direction and phase for special moves of a diffraction grating is discussed, allowing simplification in the design of the coherently tiled grating diagnostics. An analytical proof of the existence of a unique diffraction grating eigenvector for translational and rotational motion which conserves the diffraction direction and diffracted wave phase is presented.

OCIS codes: (050.1950) Diffraction gratings; (050.1960) Diffraction theory; (320.7090) Ultrafast lasers. 
Fast ignition $^{1}$ for high-yield inertial confinement fusion (ICF) represents an attractive route to enhancement of conventional ICF driven by nanosecond lasers. Recent experiments $^{2}$ suggest that the delivery of energy in excess of $100 \mathrm{~kJ}$ in $10-20$ ps pulses to the compressed target will be needed for efficient full-scale fast ignition. Production of energy on this scale utilizing a chirped-pulse amplification (CPA) laser system represents a formidable challenge. This is particularly the case due to limited apertures and energy handling capability of diffraction gratings in the pulse compressors of such laser systems. ${ }^{3}$ Coherent tiling of gratings for high energy laser pulse compression has been proposed $^{4}$ and recently demonstrated ${ }^{5}$ as a route to overcome the limited energy handling of diffraction gratings. Remaining challenges to the full adoption of this technique include the design and operation of coherently tiled grating alignment diagnostics and the interpretation of the misaligned grating diagnostics signatures. In this Letter we report the existence of a unique eigenvector for diffraction grating translational and rotational motion. Grating diffraction direction is unaffected by the grating translation in the direction parallel to, or rotation around the axis parallel to this grating eigenvector, unique to a selected diffraction order. Multiple practical design features and capabilities of the coherently tiled grating diagnostics will be enabled by taking advantage of the existence of diffraction eigenvectors.

Maintaining alignment of the multiple gratings presents a significant technological challenge in any implementation of the coherent grating tiling concept. Of the six possible rigid-body motions (three translational and three rotational) of one grating relative to another, only one, translation along the grooves, obviously does not affect the phasing of the gratings. As pointed out earlier, ${ }^{5}$ since there are only three 
possible errors in the wavefront: tip, tilt, and path length, the effect on phasing of the five remaining rigid-body motions must be coupled. This may lead to a significant reduction in the complexity of controls needed to maintain grating alignment since one motion may be used to compensate for errors in another.

Fig. 1 shows two gratings with groove spacing $d$ displaced from each other in a direction perpendicular to the grooves. The magnitude of the displacement of some corresponding point on the grooves of each grating is characterized by $p$, the amount of piston motion, and $t$, the amount of translation in the plane of the grating surface. A plane wave is incident on the gratings with a propagation direction $\Theta$ from the grating normal. As a result of the translation, the optical path difference $(O P D)$ for the light striking grating 2 compared to the path length to grating 1 is $p \cos (\Theta)-t \sin (\Theta)$. Identical analysis can be done to find the $O P D$ for the diffracted wave. Using the sign convention that a diffracted angle on the opposite side of the normal from a positive incidence angle is positive and denoting the diffracted angle by $\Theta^{\prime}$, the total $O P D$ due to the translation is

$$
O P D=p\left[\cos (\Theta)+\cos \left(\Theta^{\prime}\right)\right]-t\left[\sin (\Theta)-\sin \left(\Theta^{\prime}\right)\right] .
$$

The diffraction from a pair of gratings displaced with respect to each other is identical to two perfectly aligned gratings when the $O P D$ equals an integral number of waves. Without loss of generality we can consider alignment to be achieved when $O P D=0$. Adding an integral number of waves corresponds to shifting the second grating by an integral number of grooves. The gratings will remain phased provided the translation direction satisfies

$$
\frac{p}{t}=\frac{\sin (\Theta)-\sin \left(\Theta^{\prime}\right)}{\cos (\Theta)+\cos \left(\Theta^{\prime}\right)}=\tan \left(\frac{\Theta-\Theta^{\prime}}{2}\right) .
$$


Eq. (2) shows that the motion that results in zero $O P D$ is a translation perpendicular to the bisector of the input and diffracted wave vectors. Thus the translational eigenvector for a diffraction grating is parallel to the bisector of the input and diffracted wave vectors. As a consequence of this result and the invariance of the system to translation along the grating grooves, only one of the three rigid-body translations contributes to the $O P D$ at a single wavelength. Any translational motion which is not parallel to either of the motions that leave the $O P D$ invariant can be used to control the $O P D$ in a phased grating system. In practice, the piston, $p$, would seem to be the easiest variable to control.

We next consider the rotational eigenmotion of the diffraction grating. Rotation of the grating leads to conical diffraction. The vector form of the grating equation is used in this analysis ${ }^{6}$ for treatment of general conical diffraction. After preliminaries to establish notations, the existence of the rotational eigenmotion is demonstrated. It is shown that the rotational eigenvector is identical to the translational eigenvector defined by Eq. (2), i. e. parallel to the bisector of the input and diffracted wave vectors. The grating equation in vector form is ${ }^{6}$

$$
\left(\mathbf{S}-\mathbf{S}^{\prime}\right) \times \mathbf{N}=m \frac{\lambda}{d} \mathbf{G} .
$$

The notation is explained in Fig. 2. $\mathbf{N}$ and $\mathbf{G}$ are unit vectors fixed to the grating and in the direction of the outward normal and along the grooves, respectively. The unit vector in the plane of the grating and normal to the grooves, $\mathbf{P}=\mathbf{N} \times \mathbf{G}$, is also needed for the analysis. The vectors $\mathbf{S}$ and $\mathbf{S}^{\prime}$ are the unit vectors in the direction of the incident and diffracted waves, respectively. Several other mutually orthogonal sets of unit vectors will be used in the subsequent development. Let us define $\Lambda$ as the unit vector parallel to the bisector of the angle between the incident and diffracted wave vectors, i.e. the grating 
translation eigenvector. The grating is rotated by an angle $\Phi$ about the rotation axis parallel to $\Lambda$. A coordinate system independent of $\Phi$ which coincides with $(\mathbf{P}, \mathbf{N}, \mathbf{G})$ when $\Phi=0$ is denoted by $(\mathbf{X}, \mathbf{Y}, \mathbf{Z})$. The incident wave direction does not rotate and is in the $(\mathbf{X}, \mathbf{Y})$ plane. At $\Phi=0$, the diffracted wave direction is also the $(\mathbf{X}, \mathbf{Y})$ plane. It is be helpful to define a coordinate system $\left(\mathbf{X}^{\prime}, \mathbf{Y}^{\prime}, \mathbf{Z}\right)$ which is generated from the $(\mathbf{X}, \mathbf{Y}, \mathbf{Z})$ system by rotating about the $\mathbf{Z}$ axis to bring the $\mathbf{Y}$ axis into the rotation axis. It can then be seen that the unit vectors in this system are $\left(\mathbf{X}^{\prime}, \mathbf{Y}^{\prime}, \mathbf{Z}\right)$ where

$$
\mathbf{Y}^{\prime}=\frac{\mathbf{S}-\mathbf{S}^{\prime}}{\sqrt{2\left(1-\mathbf{S} \cdot \mathbf{S}^{\prime}\right)}} \text { and } \mathbf{X}^{\prime}=\mathbf{Y}^{\prime} \times \mathbf{Z} \text {. }
$$

Since $\mathbf{G}=\mathbf{Z}$ when $\Phi=0$, it is readily seen that

$$
\mathbf{G}=\mathbf{Z} \cos (\Phi)+\mathbf{X}^{\prime} \sin (\Phi) .
$$

It can be also shown that

$$
\begin{aligned}
& \mathbf{N}=\mathbf{Y}^{\prime}\left(\mathbf{Y}^{\prime} \cdot \mathbf{Y}\right)+\left(\mathbf{Y}^{\prime} \times \mathbf{G}\right)\left(\mathbf{X}^{\prime} \cdot \mathbf{Y}\right) \\
& \mathbf{P}=\left(\mathbf{Y}^{\prime} \times \mathbf{G}\right)\left(\mathbf{Y}^{\prime} \cdot \mathbf{Y}\right)-\mathbf{Y}^{\prime}\left(\mathbf{Y}^{\prime} \cdot \mathbf{X}\right)
\end{aligned}
$$

With the notation established, the proof that rotation about the bisector of the incident and diffracted wave directions leaves the diffracted wave direction unchanged is straightforward. Taking the scalar product of Eq. (6) with $\mathbf{P}$ gives

$$
\mathbf{G} \cdot\left(\mathbf{S}-\mathbf{S}^{\prime}(\Phi)\right)=0 .
$$

From the definition of $\mathbf{Y}^{\prime}$ and Eq. (8a), it can also be seen that

$$
\mathbf{G} \cdot\left(\mathbf{S}-\mathbf{S}^{\prime}(0)\right)=0,
$$

and therefore

$$
\mathbf{G} \cdot\left(\mathbf{S}^{\prime}(\Phi)-\mathbf{S}^{\prime}(0)\right)=0 .
$$


Next, take the scalar product of Eq. (6) with $\mathbf{G}$ to get

$$
\mathbf{P} \cdot\left(\mathbf{S}-\mathbf{S}^{\prime}(\Phi)\right)=m \frac{\lambda}{d} .
$$

From Eqs. (6), (7), and (8b) we can also find

$$
\mathbf{G} \cdot\left(\mathbf{S}-\mathbf{S}^{\mathbf{\prime}}(0)\right)=m \frac{\lambda}{d},
$$

and therefore

$$
\mathbf{P} \cdot\left((\Phi)-\mathbf{S}^{\prime}(0)\right)=0 .
$$

Now according to Eqs. (11) and (14), the two unit vectors $\mathbf{S}^{\prime}(\Phi)$ and $\mathbf{S}^{\prime}(0)$ have identical components in each of two mutually orthogonal directions. This requires that the two unit vectors are equal. Thus the invariance is proven. Of the three independent rigid-body rotations, one, rotation about the bisector of the incident and diffracted rays, does not affect the diffracted wavefront. Therefore, similar to the situation in the previous section, any two rotations not parallel to this eigenmotion need to be controlled in order to phase the gratings at one wavelength. Experimental mounting a $600-\mathrm{mm}^{-1}$ grating and the use of a 543-nm laser beam confirmed the existence of the grating rotation eigenvector.

It has been shown above that the coupling of degrees of freedom of the grating motion may be expressed very intuitively in terms of two 'eigenmotions' of the relative positions of the gratings that leave the diffracted waves phased. The two eigenmotions (translational and rotational) share the identical eigenvector, which is defined by the bisector of the incident and diffracted wave vectors. Only the three degrees of freedom orthogonal to these eigenmotions must be controlled to maintain coherence between the gratings for a monochromatic beam. However, these eigenmotions depend both on the wavelength and the incidence angle of the incoming light and this has significant 
implications for the application of phased gratings to CPA systems. The wavelength dependence means that there is no one eigenmotion that will maintain the phasing over the entire bandwidth of the chirped pulse. The dependence on angle of incidence implies that any diagnostic that utilizes an alignment laser must operate at the same angle of incidence as the high-energy chirped-pulse beam. If the diagnostic beam does not share the grating eigenvector with the use beam, the motions for which the diagnostic is not sensitive will affect the use beam. The effect of the spectral bandwidth, diagnostic beam direction, and differences in groove density on grating tiling will be the subject of the forthcoming analysis.

This work was performed under the auspices of the U. S. Department of Energy by the University of California, Lawrence Livermore National Laboratory under Contract No. W-7405-Eng-48. 


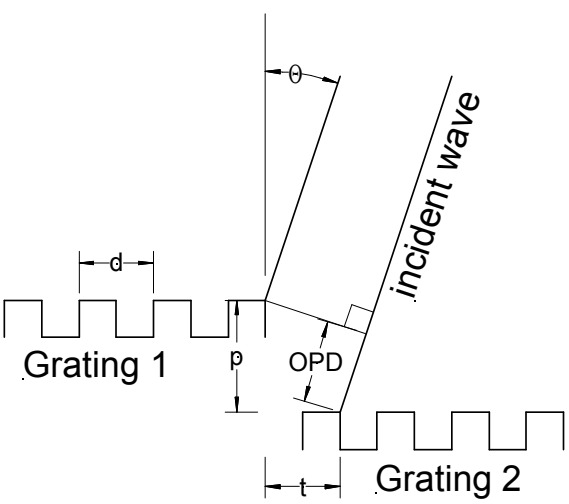

Figure 1 

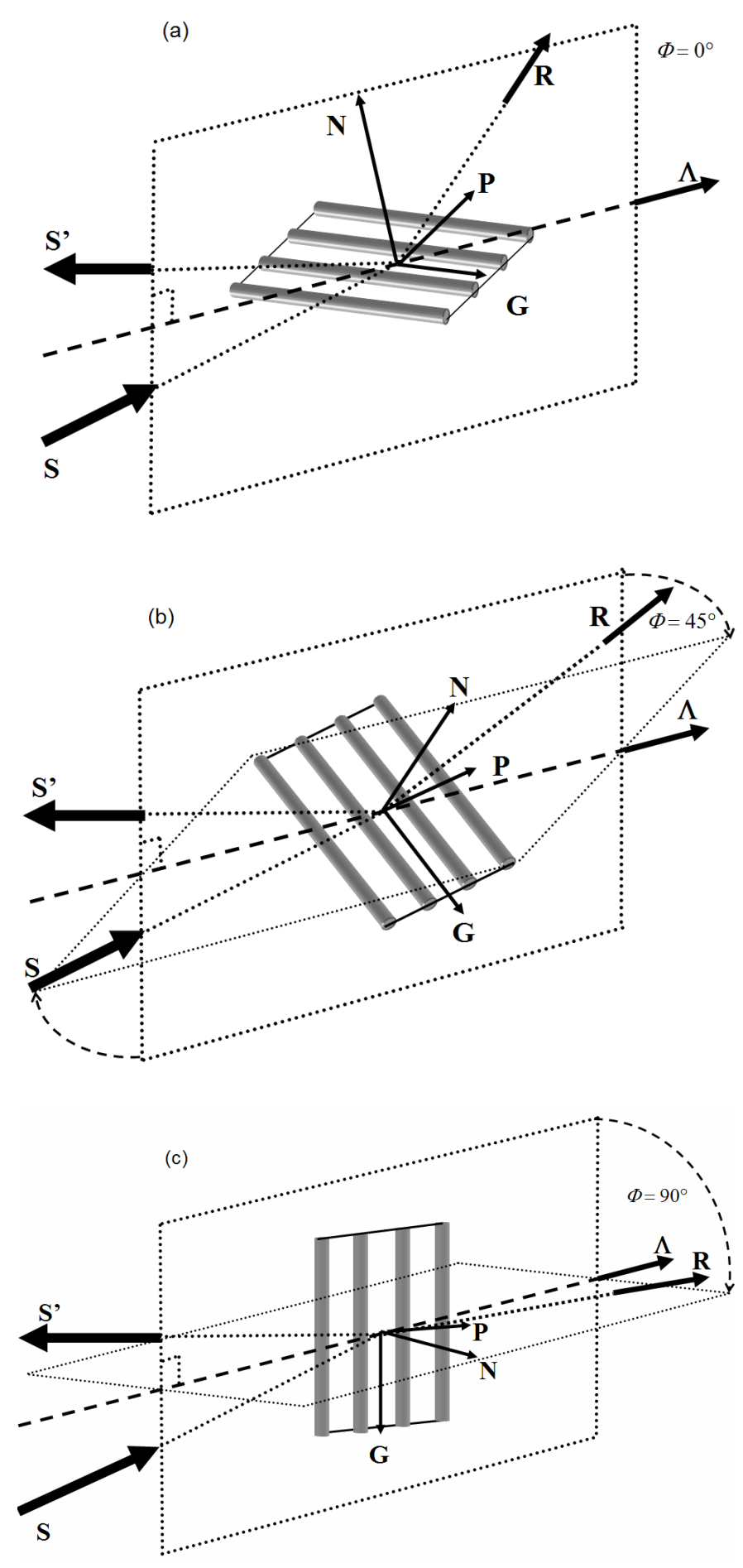

Figure 2 


\section{LIST OF FIGURES}

Figure 1. OPD for a wave incident at an angle $\Theta$ on a set of parallel gratings displaced by the piston motion $p$ and lateral motion $t$. The two gratings are assumed to have identical grating period $d$.

Figure 2. (a) Notation for grating conical diffraction analysis. The unit vector normal to the grating surface is denoted by $\mathbf{N}$, while the unit vector along the grooves is denoted by $\mathbf{G}$. The unit vector in the plane of grating and normal to the grooves is denoted by $\mathbf{P}$. The incident and diffracted wave unit vectors are $\mathbf{S}$ and S', respectively. The reflection vector is denoted by $\mathbf{R}$. In (b) and (c) we show the rotation of the grating about the grating eigenvector $\Lambda$ by $45^{\circ}$ and $90^{\circ}$, respectively. 


\section{LIST OF REFERENCES}

1. M. Tabak, J. Hammer, M.E. Glinsky, W.L. Kruer, S.C. Wilks, J. Woodworth, E.M. Campbell, M.D. Perry, and R.J. Mason, Ignition and High-Gain with Ultrapowerful Lasers. Phys. Plasmas 1, 1626 (1994).

2. R. Kodama, P.A. Norreys, K. Mima, A.E. Dangor, R.G. Evans, H. Fujita, Y. Kitagawa, K. Krushelnick, T. Miyakoshi, N. Miyanaga, T. Norimatsu, S.J. Rose, T. Shozaki, K. Shigemori, A. Sunahara, M. Tampo, K.A. Tanaka, Y. Toyama, Y. Yamanaka, and M. Zepf, Fast heating of ultrahigh-density plasma as a step towards laser fusion ignition. Nature 412, 798 (2001).

3. C.P.J. Barty, M. Key, J. Britten, R. Beach, G. Beer, C. Brown, S. Bryan, J. Caird, T. Carlson, J. Crane, J. Dawson, A.C. Erlandson, D. Fittinghoff, M. Hermann, C. Hoaglan, A. Iyer, L. Jones, I. Jovanovic, A. Komashko, O. Landen, Z. Liao, W. Molander, S. Mitchell, E. Moses, N. Nielsen, H.H. Nguyen, J. Nissen, S. Payne, D. Pennington, L. Risinger, M. Rushford, K. Skulina, M. Spaeth, B. Stuart, G. Tietbohl, and B. Wattellier, An overview of LLNL high-energy short-pulse technology for advanced radiography of laser fusion experiments. Nuclear Fusion 44, S266 (2004).

4. T.J. Zhang, M. Yonemura, and Y. Kato, An array-grating compressor for highpower chirped-pulse amplification lasers. Opt. Commun. 145, 367 (1998).

5. T.J. Kessler, J. Bunkenburg, H. Huang, A. Kozlov, and D.D. Meyerhofer, Demonstration of coherent addition of multiple gratings for high-energy chirpedpulse-amplified lasers. Opt. Lett. 29, 635 (2004). 
6. G.H. Spencer and M. Murty, General ray-tracing procedure. J. Opt. Soc. Am. 52, $672(1962)$. 